\title{
Manejo de enfermedad vascular oclusiva mediante trombectomía mecánica y/o aspiración
}

\author{
Management of occlusive vascular disease by mechanical thrombectomy and/or \\ aspiration
}

Gestão da doença vascular oclusiva por trombectomia mecânica e / ou aspiração

Jimmy Achi Arteaga a , Cintya Rodríguez Bosquez ${ }^{\text {b }}$, Janina Cueva Ludeña (D) c

\begin{abstract}
a Centro de Estudios de Enfermedades Neurológicas, Servicio de Neurocirugía, Hospital Clínica Guayaquil, Guayaquil, Ecuador; b Centro de Estudios de Enfermedades Neurológicas, Guayaquil, Ecuador; c Servicio de Cirugía General, Hospital Clínica Guayaquil, Universidad de Especialidades Espíritu Santo, Guayaquil, Ecuador.
\end{abstract}

Correspondencia a:

Janina Cueva Ludeña,

janincueval@gmail.com

Recibido: 15 de octubre, 2020

Aceptado: 17 de noviembre, 2020

Publicado: 30 de diciembre, 2020

\section{ARTÍCULO ORIGINAL}

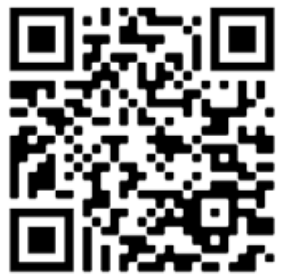

Escanea en tu dispositivo móvil o revisa este artículo en: https:// revistaclinicaguayaquil.org

\section{RESUMEN}

Introducción: El manejo de las patologías vasculares cerebrales ha estado en constante evolución durante los últimos años y, en la actualidad, la cirugía endovascular se ha convertido en una herramienta valiosa para el tratamiento y manejo de las mismas.

Materiales y Métodos: Estudio retrospectivo y analítico de una serie de 96 pacientes consecutivos, en el período comprendido entre 2013 y 2020, con diagnóstico de ictus isquémico a los que se le realizó trombectomía mecánica. Se utilizó el dispositivo de recanalización tipo Solitaire ${ }^{\circledR}$ asociado a rtPA intraarterial $10 \%$ de la dosis intravenosa.

Resultados: Se obtuvo una recanalización en el 95\% de los pacientes, la efectividad del tratamiento fue evaluada con la escala de Rankin modificada con un resultado de: 31 pacientes (32.3\%) con Rankin 0; 40 pacientes (41.7\%) con discapacidad leve aislada entrando en la categoría de Rankin 1; 11 pacientes (11.5\%) con Rankin 2; 4 pacientes (4.1\%) con Rankin 3, ya que presentaron una complicación con transformación a hemorrágica; sin recuperación alguna de su sintomatología (Rankin 5) un total de 6 pacientes (6.3\%) y, dentro del Rankin 6, es decir muerte: 4 pacientes $(4,1 \%)$.

Conclusiones: Los resultados del presente estudio son alentadores, sobre todo al reducir la secuela discapacitante en esta población.

Palabras clave: Ictus; trombectomía mecánica; escala de Rankin modificada

\footnotetext{
ABSTRACT

Introduction: During the last years, the management of cerebral vascular pathologies has been in constant evolution. Nowadays, endovascular surgery has become a very valuable tool for their treatment and management.

Materials and Methods: A retrospective and analytical study was conducted on a series of 96 consecutive patients, between 2013 and 2020, diagnosed with ischemic stroke who underwent mechanical thrombectomy with the Solitaire ${ }^{\circledR}$ recanalization device associated with intra-arterial rtPA 10\% of the intravenous dose was used.

Results: Recanalization was obtained in $95 \%$ of the patients, the effectiveness of the treatment was evaluated with the modified Rankin scale with the following results: 31 patients (32.3\%) with Rankin score 0; 40 patients $(41.7 \%)$ with isolated mild disability, corresponding to the Rankin 1 category; 11 cases (11.5\%) with Rankin score of 2; 4 patients (4.1\%) with Rankin 3 score, as they presented transformation to hemorrhagic disease as a complication; a total of 6 cases (6.3\%) without any recovery of their symptoms (Rankin 5) and, within Rankin 6 category, corresponding to patients who died: 4 cases $(4.1 \%)$.
} 
Conclusions: The results of the present study are encouraging, especially reducing disabling sequelae in this population.

Key words: Ictus; mechanical thrombectomy; modified Rankin scale.

\section{RESUMO}

Introdução: A gestão das patologias vasculares cerebrais tem estado em constante evolução nos últimos anos e, na atualidade, a cirurgia endo vascular tem-se tornado uma ferramenta muito valiosa para o tratamento e gestão.

Materiais e Métodos: Estudo retrospectivo e analítico de uma série de 96 pacientes consecutivos, no período de 2013 até 2020, com diagnóstico de acidente vascular cerebral isquêmico que foram submetidos à trombectomia mecânica. Foi utilizado dispositivo de recanalização tipo Solitaire $\circledast$ associado a rtPA intra-arterial, $10 \%$ da dose intravenosa.

Resultados: A recanalização foi obtida em $95 \%$ dos pacientes, a eficácia do tratamento foi avaliada com a escala de Rankin modificada, obtendo os seguintes resultado: 31 pacientes (32,3\%) com Rankin 0; 40 pacientes $(41,7 \%)$ com deficiência leve isolada entrando na categoria Rankin 1; 11 pacientes (11,5\%) com Rankin 2 ; 4 pacientes $(4,1 \%)$ com Rankin 3, já que apresentavam complicação com transformação para hemorrágica; sem qualquer recuperação dos sintomas, ou seja, Rankin 5, um total de 6 pacientes $(6,3 \%)$ e, dentro do Rankin 6 , ou seja, óbito: 4 pacientes $(4,1 \%)$.

Conclusões: Os resultados do presente estudo são animadores, principalmente na redução das sequelas incapacitantes nesta população.

Palavras-chave: AVC; trombectomia mecânica; escala de Rankin modificada

\section{INTRODUCCIÓN}

La enfermedad cerebrovascular (ECV) isquémica aguda es una de las causas más frecuentes de discapacidad permanente y muerte en los países desarrollados y en vías de desarrollo. En las últimas décadas se han creado diversas guías para su manejo, diagnóstico y tratamiento oportuno, con el fin de corregir y evitar secuelas neurológicas irreversibles, aun así y a pesar de los esfuerzos, esta sigue alcanzando tasas elevadas de morbimortalidad (1-3).

Los pacientes con ECV isquémica tienen un mayor riesgo de mortalidad por causas a la edad avanzada, presencia de insuficiencia renal crónica, dislipidemias, antecedentes de insuficiencia cardiaca, fibrilación auricular, presentación con hemiplejía, y los signos de isquemia aguda (edema perilesional) en la tomografía de cerebro $(2,4)$. Los ECV anteriores, así como ataques isquémicos transitorios (AIT) son predictores de mortalidad; se han asociado a un mejor pronóstico, la afectación del territorio de la arteria cerebral media derecha y en aquellos pacientes que reciben tratamiento con estatinas (3).

Dentro de la clasificación del ictus se incluye el aterotrombótico, cardioembólico, lacunar e indeterminado. Las guías del manejo de ECV isquémico indican como tratamiento estándar, para los pacientes que se presentan dentro de las 4 a 5 horas desde el inicio de los síntomas, la trombólisis intravenosa, evaluando el caso para descartar contraindicaciones como la anticoagulación oral, cáncer o cirugía reciente $(2,3,5)$.

El objetivo de este manuscrito es describir una serie de casos de pacientes con oclusiones arteriales, las cuales fueron resueltas mediante trombectomía mecánica, con la finalidad de representar las ventajas, seguridad y posibles limitaciones de la misma.

\section{MATERIALES Y MÉTODOS}

Se trata de un análisis retrospectivo de una serie de 96 pacientes consecutivos con diagnóstico de accidente cerebrovascular isquémico agudo, los cuales se sometieron a trombectomía mecánica en un período de tiempo comprendido entre julio del año 2013 y agosto de 2020. En todos los pacientes se realizó un protocolo previo a la cirugía que incluía: historia clínica neurológica, angio-tomografía, angio-resonancia y exámenes pre-operatorios.

En los criterios de inclusión fueron tomados en consideración los siguientes:

Edad mayor de 18 años.

- Tomografía de cerebro simple sin signos de lesiones isquémicas o hemorrágicas agudas al ingreso.

- La ventana terapéutica incluyó los pacientes con menos de 8 horas de evolución desde el inicio de los síntomas, para los de circulación anterior, y menor de 12 horas, en territorio vertebro basilar.

- En la estadificación se utilizó la escala NIHSS (National Institute of Health Stroke Scale) de 15 - 20 puntos.

Los pacientes fueron trasladados a la sala de angiología, en donde se realizó el diagnóstico angiográfico, comprobando la oclusión vascular. Posterior a esto se inyectó rtPA intraarterial, el $10 \%$ de la dosis administrada IV, previo el uso del dispositivo de revascularización Solitaire ${ }^{\mathrm{TM}}$ FR (Figura 1). Se realizaron imágenes pre y post trombectomía (Figuras 2 y 3 ). 


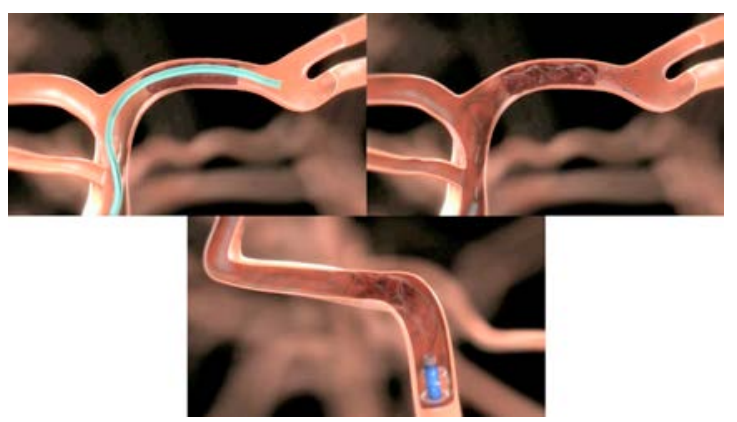

Figura 1. Dispositivo de revascularización Solitaire ${ }^{\mathrm{TM}} \mathrm{FR}$, reproducido de: Covidien. Nuevo estudio demuestra que la adición de trombectomía mecánica mediante stent resulta más eficaz que el tratamiento farmacéutico tradicional en pacientes con accidente cerebrovascular isquémico agudo. BusinessWire. 2014.

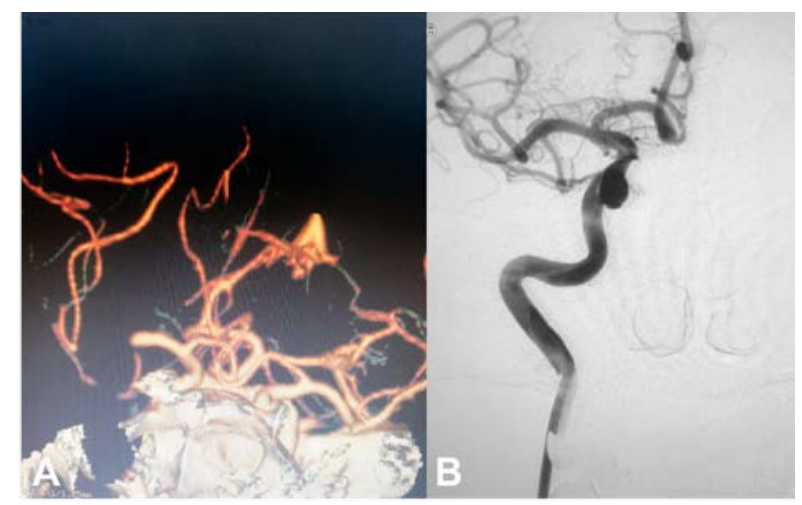

Figura 2. A. Circulación anterior: oclusión de arteria cerebral media derecha. B. Recanalización de la arteria cerebral media derecha.

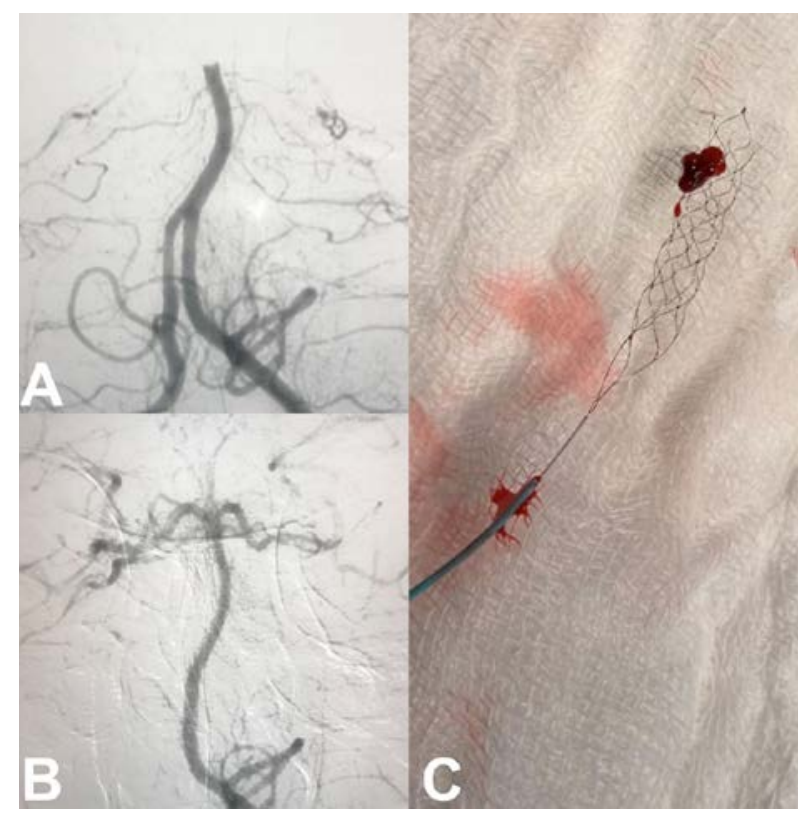

Figura 3. A. Circulación posterior: oclusión de la arteria basilar. B. Recanalización de la arteria basilar. C. Dispositivo para trombectomía mecánica SolitaireTM.

\section{RESULTADOS}

Un total de 96 pacientes fueron incluidos en el estudio, de los cuales 89 (92.7\%) pertenecían al género masculino y 7 (7.3\%) al femenino. Clasificándolos de acuerdo a su localización, se obtuvo un total de 85 pacientes (88.5\%) con

afectación en la circulación anterior (Figura 2) y 11 pacientes (11.5\%) con afectación en la circulación posterior (Figura 3, Tabla 1). Con respecto a la sintomatología presentada al momento del ingreso, el $89 \%$ presentó hemiplejia unilateral y el $11 \%$ afasia.

Tabla1. Características demográficas generales.

Características

\section{Sexo}

Masculino

Femenino

$7(7.3)$

\section{Localización}

Circulación anterior

Circulación posterior

$11(11.5)$

\section{Dispositivo utilizado}

Solitaire ${ }^{\mathrm{TM}} \mathrm{FR}$ 96 (100)

La efectividad terapéutica se evaluó mediante la escala de Rankin modificada al alta de cada paciente. Se logró una recanalización imagenológica posterior al procedimiento en el $95 \%$ de los casos. De esta forma, se obtuvo un total de 31 pacientes (32.3\%) con recuperación completa de sus funciones (Rankin: 0); 40 pacientes (41.7\%) con discapacidad leve aislada, correspondiendoalacategoría de Rankin 1 , siendo la más frecuente la parálisis facial; 11 pacientes (11.5\%) con Rankin 2, ya que presentaron paresia leve que les permitía independencia de sus actividades; 4 pacientes (4.1\%) con score de Rankin 3, quienes presentaron como complicación la transformación hemorrágica del infarto, 24 horas posterior al procedimiento, el mismo que requirió cirugía, quedando como secuela una paresia moderada; un total de 6 pacientes (6.3\%) no obtuvo recuperación alguna de su sintomatología (score de Rankin 5) y, dentro del grupo de Rankin 6, es decir muerte, se encontraron 4 pacientes $(4,1 \%)$ (Figura 4, Tabla 2).

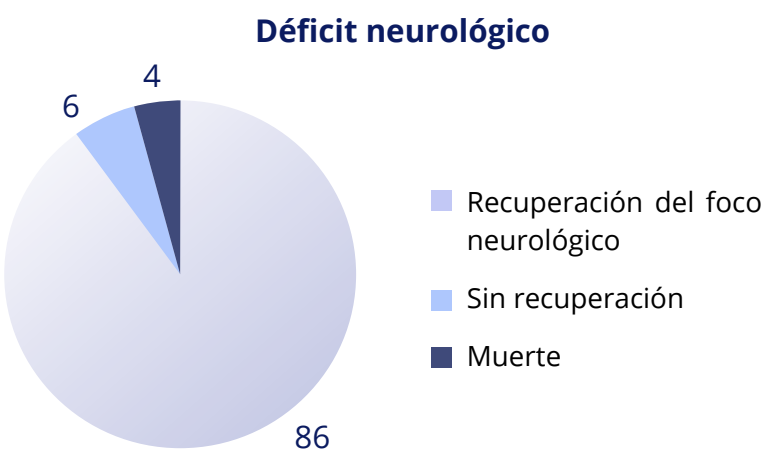

Figura 4. Resultados del déficit neurológico. 
Tabla 2. Resultados correlacionados con la escala modificada de Rankin.

\begin{tabular}{cc}
\hline Estatificación & n (\%) \\
Rankin 0 & $31(32.3)$ \\
Rankin 1 & $40(41.7)$ \\
Rankin 2 & $11(11.5)$ \\
Rankin 3 & $4(4.1)$ \\
Rankin 4 & $0(0)$ \\
Rankin 5 & $6(6.3)$ \\
Rankin 6 & $4(4.1)$ \\
\hline
\end{tabular}

Como resultado se obtuvo una mejoría significativa en el $89 \%$ de los pacientes, comparable con estudios similares, con una mejoría a 90 días evaluada mediante la escala de Rankin modificada, de 0 a 2 puntos.

\section{DISCUSIÓN}

La isquemia cerebral aguda (ictus) se considera una urgencia médica. Se debe principalmente, a que los mecanismos lesionales que se desencadenan una vez ocurrida la isquemia progresan rápidamente, y es corto el período durante el cual los tratamientos aplicados pueden ser efectivos (6). La trombectomía mecánica endovascular ha mostrado ser eficaz y segura, pudiendo obviar o complementar la necesidad de utilizar agentes trombolíticos que aumentan el riesgo de hemorragia. Según las guías de Stroke publicadas en el 2018, se estableció un margen más amplio de tiempo, que se prolonga, incluso, hasta 24 horas después de presentar un ictus en pacientes cuidadosamente seleccionados, aumentando en más del $60 \%$ las probabilidades de recuperación neurológica y la proporción de pacientes que pueden beneficiarse de esta intervención (7). Las indicaciones de la técnica están establecidas, se tiene que individualizar cada caso, y evaluar si la situación clínica del paciente se puede beneficiar del procedimiento $(8,9)$.

Se han desarrollado diversos estudios, con alto nivel de confianza, en relación a la terapia trombolítica en ECV isquémico para el uso de rtPA (NINDS 1 y 2, ECASS, ATLANTIS STUDY, ECASS II), sin embargo, su uso siendo controvertido, particularmente, porque no está claro si una terapia que dependa del tiempo, la tecnología y la infraestructura se puede aplicar de manera amplia y segura $(5,6,10)$. En la actualidad, la recanalización mecánica es una opción de tratamiento prometedor para pacientes con oclusión de las arterias cerebrales como causa de ECV isquémico, y es usada frecuentemente como terapia conjunta a la terapia trombolítica intravenosa como "bridging therapy" $(3,5,6,8)$.

La trombectomía mecánica, como técnica de primera elección, es una terapéutica útil en nuestro medio para el manejo de pacientes con ECV isquémico agudo, dando la oportunidad de recuperar tejido cerebral viable y disminuir la secuela neurológica del mismo. Dentro de las limitaciones para realizar este procedimiento se encuentran el diagnóstico tardío de la patología, el manejo prehospitalario inadecuado, y el alto costo del procedimiento, limitando el acceso al mismo (7). En cuanto a las complicaciones son similares a las presentadas en otras series.

\section{CONCLUSIONES}

La trombectomía mecánica es una opción de tratamiento que permite mejorar la sintomatología $y$, sobretodo, reducir la discapacidad en aquellos pacientes que acuden al hospital dentro del período de ventana y que no son candidatos, por diversas situaciones, a la terapia trombolítica intravenosa.

\section{REFERENCIAS BIBLIOGRÁFICAS}

1. Mérida-Rodrigo L, Poveda-Gómez F, CamafortBabkowski M, Rivas-Ruiz F, Martín-Escalante MD, Quirós-López R, et al. Supervivencia a largo plazo del ictus isquémico. Rev Clínica Española. 2012; 212(5):223-8.

2. Castaño C, Dorado L, Guerrero C, Millán M, Gomis M, Pérez De La Ossa N, et al. Mechanical thrombectomy with the solitaire $A B$ device in large artery occlusions of the anterior circulation: A pilot study. Stroke. 2010; 41(8):1836-40.

3. Hill MD, Buchan AM, Investigators CA for SES (CASES). Thrombolysis for acute ischemic stroke: results of the Canadian Alteplase for Stroke Effectiveness Study. CMAJ. 2005 May 10; 172(10):1307-12.

4. Sczesni K, Wiebringhaus R, Heuser L, Skodda $\mathrm{S}$, Eyding J. Mechanical thrombectomy - An alternative treatment option in a patient with acute ischemic stroke and multiple contraindications for systemic thrombolysis: A case report. J Med Case Rep. 2013 Nov 7; 7: 256.

5. Hacke W, Donnan G, Fieschi C, Kaste M, von Kummer R, Broderick JP, et al. Association of outcome with early stroke treatment: pooled analysis of ATLANTIS, ECASS, and NINDS rt-PA stroke trials. Lancet. 2004 Mar; 363(9411):768-74.

6. Rengel MD, Gil Romero J, De Freytas Rodriguez A, Sanchis García JM, Guijarro Rosaleny J, Palmero Da Cruz J. Trombectomía mecánica en el ictus: análisis retrospectivo en un año de experiencia. Interv. 2018; 18(3):89-97.

7. McCoy CE, Langdorf Ml, Lotfipour S. American 
Heart Association/American Stroke Association Deletes Sections from 2018 Stroke Guidelines. West J Emerg Med. 2018 Nov; 19(6):947-51.

8. Carter AM, Catto AJ, Mansfield MW, Bamford JM, Grant PJ. Predictive variables for mortality after acute ischemic stroke. Stroke. 2007; 38(6):187380.

9. Campodónico O. D, López R. A, San Román M. L,
Blasco A. J, Oleaga Z. L, Macho F. J. Trombectomía mecánica en el ICTUS; Experiencia con trevo en hospital provincial clínico Barcelona. Rev Chil Radiol. 2013; 19(2):60-3.

10. Montaner J, Álvarez-Sabín J. La escala de ictus del National Institute of Health (NIHSS) y su adaptación al español. Neurología. 2006; 21:192202. 


\section{ACERCA DE LOS AUTORES}

1. Jimmy Achi Arteaga: Staff de neurocirugía en el Centro de Estudios de Enfermedades Neurológicas y Hospital Clínica Guayaquil; Guayaquil, Ecuador

2. Cintya Rodríguez Bosquez: Médico graduado de la Universidad Católica Santiago de Guayaquil. Centro de Estudios de Enfermedades Neurológicas, Guayaquil, Ecuador.

3. Janina Cueva Ludeña: Médico Posgradista de Cirugía General, Universidad de Especialidades Espíritu Santo, Hospital Clínica Guayaquil, Guayaquil, Ecuador.

ORCID: 0000-0003-1145-7455 